\title{
Author Correction: A needle for Alzheimer's in a haystack of claims data
}

Emre Guney $(\mathbb{D}$ and Alejandro Athie

Correction to: Nature Aging https://doi.org/10.1038/s43587-021-00139-y, published online 10 December 2021.

In the version of this article initially published, the wrong journal name was listed in ref. 8. The correct ref. should read: Aguirre-Plans, J. et al. Pharmaceuticals 11, 61 (2018).

The change has been made in the html and PDF versions of the article.

Published online: 11 January 2022

https://doi.org/10.1038/s43587-022-00168-1

(c) Springer Nature America, Inc. 2022 